\title{
LEVANTAMENTO DE ABELHAS EUGLOSSINI NA CHAPADA DIAMANTINA COMO ESTRATÉGIA PARA MONITORAMENTO E CONSERVAÇÃO DAS POPULAÇÕES DE ABELHAS DE ORQUÍDEAS
}

\author{
Guilherme Pereira de Jesus ${ }^{1}$; Cândida Maria Lima Aguiar²; William Moura \\ Aguiar $^{3}$ \\ 1. Bolsista PROBIC/UEFS, Graduando em Bacharelado em Ciências Biológicas, Universidade Estadual de Feira de \\ Santana, e-mail: guidspj@gmail.com \\ 2. Orientador, Departamento de Ciências Biológicas, Universidade Estadual de Feira de Santana, e-mail: \\ candida.aguiar@gmail.com \\ 3. Participante do projeto, Departamento de Ciências Biológicas, Universidade Estadual de Feira de Santana, e-mail: \\ wmag26@yahoo.com
}

PALAVRAS-CHAVE: ecologia; comunidade; inseto.

\section{INTRODUÇÃO}

A tribo Euglossini consiste em um grupo de abelhas de distribuição predominantemente neotropical que tem como característica mais marcante a coleta de odores florais realizada pelos machos. Este comportamento torna os machos potencialmente polinizadores e media sua relação com muitas espécies de plantas, com destaque para a família Orchidaceae, onde mais de 700 espécies dependem destes insetos para completar seu ciclo ( Roubik \& Hanson, 2004). São capazes de voar longas distâncias (Janzen, 1971), com algumas espécies cruzando fragmentos e corpos d'água (Tonhasca et al., 2003), sendo por isso importantes na manutenção do fluxo gênico entre populações de plantas, em especial as com distribuição esparsa. Devido a essas características, sua conservação é relevante para a saúde das comunidades com que interagem, pois redução no tamanho efetivo de suas populações pode levar a aumento na endogamia em espécies vegetais, incrementando a susceptibilidade destas às intempéries ambientais e impactando na comunidade como um todo. Além disso, sua ampla distribuição, facilidade de captura utilizando armadilhas com compostos aromáticos e conspicuidade dos espécimes os tornam bons indicadores ambientais (Bonilla, 1999). Para a tomada de medidas direcionadas ao uso e conservação de Euglossini, são necessárias informações sobre a composição e variações na sua dinâmica populacional, algo deficitário dentro do cerrado. Sabe-se que as espécies da fitofisionomia consistem majoritariamente em subamostras daquelas ocorrentes em matas úmidas (Faria \& Silveira, 2011), podendo indicar maior tolerância destas em relação àquelas restritas a áreas de floresta. No entanto, a reduzida riqueza pode deverse ao baixo número de estudos, pois espécies novas continuam sendo registradas (Morato, 2014). A maioria dos levantamentos concentra-se em Minas Gerais (Ferreira, 2007; Freitas, 2009; Silveira, 2015), com apenas um de extensa duração realizado na Bahia (Aguilar, 1990), cujos dados não foram publicados. Com isso, o presente estudo objetiva levantar dados acerca da abundância, riqueza e sua variação ao longo do ano em área restrita do bioma cerrado e floresta semidecídua na Chapada Diamantina, de modo a subsidiar o monitoramento destas populações e elaboração de políticas conservacionistas para a área. 


\section{METODOLOGIA}

Amostragens foram realizadas em uma área sob Mata Estacional Semidecídua (área 1) e três pontos em fitofisionomia cerrado (área 2) no município de Lençóis, na Chapada Diamantina. As coletas foram feitas durante dois dias a cada dois meses, de agosto de 2015 a março de 2016, usando armadilhas odoríferas propostas por (Nemésio \& Morato, 2006) que foram expostas no período de 09h00min as 16h00minh. Nas armadilhas, foram utilizados seis compostos: Eucaliptol, Eugenol, Vanilina, Cinamato de Metila, Betaianona e Salicilato de Metila, os quais não foram repostos durante a exposição. No final de cada dia, as abelhas capturadas foram retiradas e sacrificadas. Os animais foram montados a seco e identificados, sendo armazenados no Laboratório de Entomologia da Universidade Estadual de Feira de Santana (UEFS). A taxonomia segue Nemésio (2009) e Bembé (2007).

Para tornar equiparável a comparação das duas fitofisionomias, foi tirada a média de abundância de indivíduos das três áreas de cerrado. Os parâmetros avaliados para mensuração da diversidade foram riqueza, abundância e diversidade (índice de Shannon-Wiener); dominância na comunidade (N/Nmax), pelo índice de Berger-Parker e de equitabilidade (H'/Hmax). Para verificação da significância estatística da diferença encontrada, o $H^{\prime}$ foi submetido ao teste $t$ de Hutcheson (1970), cuja fórmula é $t=H^{\prime} 1-$ H'2 / V S ${ }^{2} H^{\prime} 1+S^{2} H^{\prime}$, onde H' 1 E H' 2 são os índices de Shannon- Wiener e $S^{2}$ a variância para as áreas 1 e 2 , respectivamente. $S^{\prime}$ é calculado por $S^{2} H^{\prime}=(\Sigma$ fi ln fi $-(\Sigma$ fi $\log$ fi) $2 / n) / n^{2}$.

\section{RESULTADOS E DISCUSSÃO}

Nos quatro pontos de amostragem foram capturados 233 indivíduos pertencentes a 7 espécies, todas presentes em ambas as áreas e durante todo o período de coleta. As morfoespécies Eulaema nigrita, Eulaema cingulata e Euglossa cordata foram as mais abundantes, representando $22 \%, 21 \%$ e $18 \%$ da comunidade, respectivamente. A primeira foi mais abundante nos meses correspondentes ao período úmido e a segunda, ao período seco.

Tabela 1. Numero de espécies e abundância das abelhas euglossinas (Apidae, Euglossini) em quatro áreas na Chapada Diamantina, índices de diversidade, $\left(\mathrm{H}^{\prime}\right)$, equitabilidade $\left(\mathrm{J}^{\prime}\right)$, dominância $(\mathrm{d})$ e teste $\mathrm{t}$ de Hutcheson (p) em cada área.

ÁREA 1 (cerrado)

\begin{tabular}{|c|c|c|c|c|c|c|c|c|c|c|c|c|c|c|c|c|}
\hline \multirow{2}{*}{$\begin{array}{l}\text { Pontos de } \\
\text { coleta } \\
\text { Espécies }\end{array}$} & \multicolumn{4}{|c|}{ Barranco } & \multicolumn{4}{|c|}{ Teto Verde } & \multicolumn{4}{|c|}{ Coités } & \multicolumn{4}{|c|}{ Mata } \\
\hline & A & $\mathrm{O}$ & $\mathrm{J}$ & M & A & $\mathrm{O}$ & $\mathrm{J}$ & M & A & $\mathrm{O}$ & $\mathrm{J}$ & M & A & $\mathrm{O}$ & $\mathrm{J}$ & M \\
\hline Eg. carolina & 10 & 8 & 2 & - & 2 & - & 6 & 2 & 1 & 2 & 2 & 1 & 6 & - & 1 & - \\
\hline $\begin{array}{l}\text { Eg. } \\
\text { melanotricha }\end{array}$ & 7 & 5 & - & - & 11 & 2 & 10 & 1 & 1 & - & - & - & - & 1 & - & - \\
\hline
\end{tabular}

ÁREA 2 


\begin{tabular}{|c|c|c|c|c|c|c|c|c|c|c|c|c|c|c|c|c|}
\hline Eg. securigera & 4 & - & - & - & - & - & 1 & - & 1 & 1 & - & 3 & 1 & - & - & 3 \\
\hline Eg leucotricha & 3 & 1 & - & - & 1 & 1 & - & 1 & 3 & - & - & 1 & 8 & 4 & - & 1 \\
\hline Eg. despecta & 6 & - & - & - & - & 1 & - & - & - & - & - & - & 5 & 1 & - & - \\
\hline El. nigrita & 1 & - & - & - & 9 & 2 & 13 & - & - & - & 5 & 7 & 3 & - & 9 & - \\
\hline El. cingulata & 4 & - & 3 & 2 & - & 2 & 4 & - & 1 & 1 & 3 & - & 15 & 12 & 4 & - \\
\hline Total & 35 & 14 & 5 & 2 & 23 & 8 & 34 & 4 & 7 & 4 & 10 & 12 & 38 & 18 & 14 & 5 \\
\hline Total por área & 57 & & & & 49 & & & & 33 & & & & 75 & & & \\
\hline $\mathrm{H}^{\prime}$ & 1,79 & & & & & & & & & & & & 1.61 & & & \\
\hline $\mathrm{J}^{\prime}$ & 0,46 & & & & & & & & & & & & 0,37 & & & \\
\hline d & 0,24 & & & & & & & & & & & & 0,41 & & & \\
\hline $\mathrm{p}$ & 0,13 & & & & & & & & & & & & & & & \\
\hline
\end{tabular}

Legenda: A. Agosto, O. Outubro, J. Janeiro, M. Março. Eug. Euglossa, Eul. Eulaema. H' = índice de Shannon-Wiener; J' = índice de Pielou; d = índice de Berker-Parker; $\mathrm{p}$ = significância

Quase todas as espécies amostradas são conhecidas por serem tolerantes a ambientes abertos e secos, com exceção das Euglossa leucotricha e Euglossa despecta, as quais foram as únicas que não tiveram aumento na abundância no mês de janeiro de 2016, mês seguido a período de queimadas. Estas espécies são conhecidas por serem capazes de deslocar-se através de cultura de cana-de-açúcar (Millet-Pinheiro, 2005) e pastos (Tonhasca Jr.,2005) até as armadilhas odoríferas, podendo esta aptidão ter permitido que dispersassem de fragmentos distantes até os pontos de coleta neste estudo.

As duas morfoespécies pertencentes ao gênero Eulaema, E. nigrita e E. cingulata, foram as mais abundantes, representando $26,61 \%$ e $41,33 \%$ da abundância total da área 1 (cerrado) e 2 (mata), respectivamente. Eulaema nigrita teve um grande incremento no mês de janeiro, posterior a um evento de queimada próximo a Teto Verde, passando a corresponder a $41 \%$ dos indivíduos amostrados no estudo. Esta tendência é comumente encontrada em outros trabalhos sob condições ambientais semelhantes, como outras áreas de cerrado (Silveira, 2015; Pereira, 2015) e fragmentos de mata imersos em área urbana (Nemésio, 2015), o que torna esta espécie de abelha potencial indicadora de ambientes secos e abertos.

Os compostos Eucaliptol e Eugenol atraíram o maior número de indivíduos enquanto Salicilato de Metila não foi atrativo, contrastando com coletas realizadas na Amazônia, onde o oposto ocorreu. Uma explicação para o padrão encontrado é a predominância do composto atrativo nos recursos utilizados pelas abelhas da região.

\section{CONSIDERAÇÕES FINAIS}

Não houve diferença estatisticamente significativa $(\mathrm{p}=0,13)$ entre as diversidades das amostras de abelhas do cerrado $\left(H^{\prime}=1,79\right)$ e da mata $\left(H^{\prime}=1,61\right)$ (Tabela 1), com ambas consistindo em abelhas frequentemente registradas em ambientes secos e abertos. Isso pode implicar na existência de condições inapropriadas para o estabelecimento de espécies menos tolerantes, o que indicaria possível efeito de antropização nos pontos de coleta. 


\section{REFERÊNCIAS}

AGUILAR, J.B.V. 1990. Contribuição ao conhecimento dos Euglossini (Hymenoptera:

Apidae) do estado da Bahia, Brasil. Dissertação de mestrado. Instituto de Biociências da Universidade de São Paulo, São Paulo, 96p.

BEMBÉ, B. 2005. Revision der Euglossa cordata-gruppe und untersuchungen zur funktionsmorphologie und faunistik der Euglossini (Hymenoptera, Apidae).

FERREIRA, P. E., Freitas, R. F., \& Augusto, S. C. 2007. Diversidade de Euglossini (Hymenoptera: Apidae) em áreas de cerrado do triângulo mineiro, MG. Bioscience Journal, 23.

FREITAS, R.F. 2009. Diversidade e sazonalidade de abelhas Euglossini Latreille (Hymenoptera: Apidae) em fitofisionomias do bioma Cerrado em Uberlândia, MG.

Dissertação de Mestrado, Universidade Federal de Uberlândia, Uberlândia.

HUTCHESON, K. 1970. A test for comparing diversities based on the shannon formula. Journal of Theoretical Biology. 29, 151-154.

JANZEN, D.H.1971. Euglossine bees as long-distance pollinators of tropical plants. Science 171: 203-205.

MAGURRAN, A.G.1988. Ecological diversity and it's measurement. Princenton University Press, New Jersey.

MILLET-PINHEIRO, P; Schlindwein, C. 2005. Do euglossine males (Apidae, Euglossini) leave tropical rainforest to collect fragrances in sugarcane monocultures?. Revista Brasileira de Zoologia 22.4: 853-858.

MOREIRA, E. F., das Neves, E. L., \& Viana, B. F. 2015. Ocorrência de Euglossa leucotricha (Hymenoptera, Apidae) na Chapada Diamantina, Bahia, Brasil.

NASCIMENTO, S.; Canale, G. R.; Silva, D. J. 2015. Abelhas euglossina (Hymenoptera: Apidae) associadas à monocultura de eucalipto no cerrado matogrossense.Revista Árvore, v. 39, n. 2, p. 263-273.

NEMÉSIO, A. \& E.F. Morato. 2006. The orchid-bee fauna (Hymenoptera: Apidae) of Acre state (northwestern Brazil) and a re-evaluation of euglossine bait-trapping. Lundiana 7: 59-64.

NEMÉSIO, A.; Silveira, F. A. 2009. Orchid bees (Hymenoptera: Apidae) of the Brazilian Atlantic Forest. Magnolia Press.

Nemésio, A., Santos, L. M., \& Vasconcelos, H. L. 2015. Long-term ecology of orchid bees in an urban forest remnant. Apidologie, 46(3), 359-368.

PEREIRA, S. A. N. 2015. Levantamento da fauna de abelhas no município de Monte Caramelo-MG. Revista GeTeC 4.7.

ROUBIK, D. W.; Hanson, P.E. 2004. Orchid bees of tropical America: biology and field guide." Instituto Nacional de Biodiversidad (INBio), Heredia, Costa Rica.

SILVEIRA, G. C., Freitas, R. F., Tosta, T. H., Rabelo, L. S., Gaglianone, M. C., \& Augusto, S. C. 2015. The orchid bee fauna in the Brazilian savanna: do forest formations contribute to higher species diversity?. Apidologie, 46(2), 197-208.

TONHASCA JR. A.; Albuguerque, G. S.; Blackmer J. S. 2003. Dispersal of euglossine bees between fragments of the Brazilian Atlantic Forest. Journal of Tropical Ecology 19.01: 99-102.

ZAR, J.H.1996. Biostatistical Analysis Prentice-Hall, Eryelwood Cliffs, N.J. 663pp. 
